\title{
A Meta-Analysis of Antibiotics Versus Surgery for the Treatment of Acute Appendicitis
}

\author{
Luai Fadi Madanat ${ }^{1}$, Dina Wahib Shaban ${ }^{1}$, Hamzeh Mohamad Naghawi ${ }^{1}$, Haneen Samir Saker ${ }^{1}$, \\ Moaath Alsmady ${ }^{1}$, Handan Ankarali ${ }^{2}$, Tunc Eren ${ }^{3}$, Orhan Alimoglu ${ }^{3}$ \\ ${ }^{1}$ Department of Surgery, School of Medicine, University of Jordan, Amman, Jordan \\ ${ }^{2}$ Department of Biostatistics, School of Medicine, Istanbul Medeniyet University, Istanbul, Turkey \\ ${ }^{3}$ Department of General Surgery, School of Medicine, Istanbul Medeniyet University, Istanbul, Turkey
}

Email address:

handan.ankarali@medeniyet.edu.tr (H. Ankarali)

\section{To cite this article:}

Luai Fadi Madanat, Dina Wahib Shaban, Hamzeh Mohamad Naghawi, Haneen Samir Saker, Moaath Alsmady, Handan Ankarali, Tunc Eren, Orhan Alimoglu. A Meta-Analysis of Antibiotics Versus Surgery for the Treatment of Acute Appendicitis. American Journal of Bioscience and Bioengineering. Vol. 5, No. 3, 2017, pp. 75-82. doi: 10.11648/j.bio.20170503.11

Received: July 19, 2017; Accepted: July 31, 2017; Published: August 18, 2017

\begin{abstract}
Appendectomy is the mainstay of treatment for acute appendicitis. Considering the complications of surgery, antibiotic treatment has also been gaining increasing interest in cases of acute appendicitis. This study aimed to compare the efficacy of antibiotics to surgery for acute uncomplicated appendicitis. The PubMed, Medline, Medscape and Cochrane databases were searched for studies comparing antibiotics versus surgery. The six outcome measures identified were thirty-day post-therapeutic peritonitis, length of hospital stay, prevalence of total complications, prevalence of normal appendix, prevalence of mean duration of pain and duration of disability. Five prospective RCTs with a total of 1430 patients (644 in the antibiotic group and 786 in the surgical group) were included in this study. Antibiotic treatment had a success rate of $75.3 \%$. Regarding overall mean duration of disability, the antibiotic group had a significantly shorter duration of disability than that of the surgery group $(P<0.05)$. The total number of complications in the antibiotic group was $3.6 \%$ while that of the surgical group was $11.6 \%$. The overall difference for mean duration of pain, and length of hospital stay between antibiotic therapy and surgery were not found to be statistically significant $(P>0.05)$. Although the conservative approach has a success rate lower than appendectomy, it is a possible alternative in certain clinical scenarios. Appendectomy remains the mainstay treatment for acute appendicitis. However, additional studies clarifying the certain etiologies of appendicitis that are responsive to antibiotic treatment are needed to further support its use.
\end{abstract}

Keywords: Acute, Appendicitis, Antibiotics, Surgery

\section{Introduction}

Acute appendicitis, first described by Fitz in 1886, is considered the most common cause of acute abdomen with a lifetime risk of approximately 7-8\% [1-3]. Despite the fact that the exact etiology of acute appendicitis is not well understood, the majority of hypotheses suggest luminal obstruction of the appendix as the main etiology [3].

Appendectomy has been the gold standard treatment of acute appendicitis for more than 120 years since Mc Burney first explained it in 1889 [4]. It is one of the most common operations performed on the abdomen, with mortality rates ranging from $0.07 \%$ to $0.7 \%$ [5-7].
Mc Burney additionally recognized that the clinical course of appendicitis is variable, ranging from spontaneous resolution to appendiceal perforation. Hence, highlighting the need to perform immediate appendectomy in order to reduce mortality. Despite early intervention, the postoperative complications can range from $10 \%-19 \%$ and $30 \%$ for uncomplicated and complicated acute appendicitis, respectively [8-10]. The most frequent surgical complications being wound infection and intra-abdominal adhesions depending on the type of surgery used $[11,12]$.

The conservative treatment of acute appendicitis has long been appreciated since the 1950's. In 1953, Harrison reported successfully treating 42 out of 47 patients by antibiotics [13]. 
In 1956, Coldrey conducted a study to evaluate the efficacy of antibiotics in the treatment of acute appendicitis. He treated 137 patients with acute appendicitis non-operatively [14]. In 1979, combined traditional Chinese and Western medicine were used to treat 1200 patients with acute appendicitis, of whom $94.2 \%$ were treated successfully [15].

Several studies established the effectiveness of antibiotic therapy in the treatment of other intra-abdominal inflammatory conditions such as diverticulitis [16]. Furthermore, a common pathogenesis has been suggested between acute appendicitis and diverticulitis [17]. In this sense, the role of antibiotics in the treatment of acute appendicitis may have been overlooked.

Several retrospective studies demonstrated that antibiotics could be used as the sole treatment of uncomplicated acute appendicitis [18-20]. Nonetheless, the impact of antibiotics as a primary treatment of acute appendicitis has been controversial with systematic reviews and meta-analyses presenting inconsistent results [21-24].

The aim of this meta-analysis is to conclude if antibiotics can be used as a safe alternative to appendectomy in managing acute uncomplicated appendicitis, and to present the significant differences between the two modalities of treatment.

\section{Materials and Methods}

The study was registered to the Research Registry system with the Review Registry Unique Identifying Number: review registry 202 (www.figresearchregistry.com).

\subsection{Data Sources}

Prospective RCTs were selected for this study to evaluate antibiotics versus surgery for the management of acute appendicitis. The PubMed, Medline, Medscape and Cochrane online library databases were systematically searched for articles published between 1990 and 2015. Publications in English Language were found using the following MESH phrase with Boolean operators: "Acute appendicitis AND (medical OR conservative OR non-operative OR antibiotics) AND (surgical OR appendectomy OR operative)". Citations of the chosen articles were also manually screened for further potentially relevant articles.

\subsection{Data Extraction}

Four authors independently reviewed and crosschecked abstracts derived from the electronic library databases for relevance and extracted the data. Disagreements were resolved by discussion with a fifth author who supervised this process to ensure eligibility. A final consensus was reached after verifying all the papers provided by the authors.

\subsection{Study Selection Criteria}

All prospective RCTs comparing antibiotic therapy with appendectomy for patients with suspected acute appendicitis at initial presentation were eligible for inclusion. However, the inclusion criteria limited patients' age to those older than 16 years of either gender. Studies, which only enrolled patients with known complicated appendicitis, such as phlegmon or periappendiceal abscess at time of admission, were excluded. There were no restrictions on the type of antibiotic or duration of its use. Open or laparoscopic appendectomy was performed at the surgeon's preference (Figure 1).

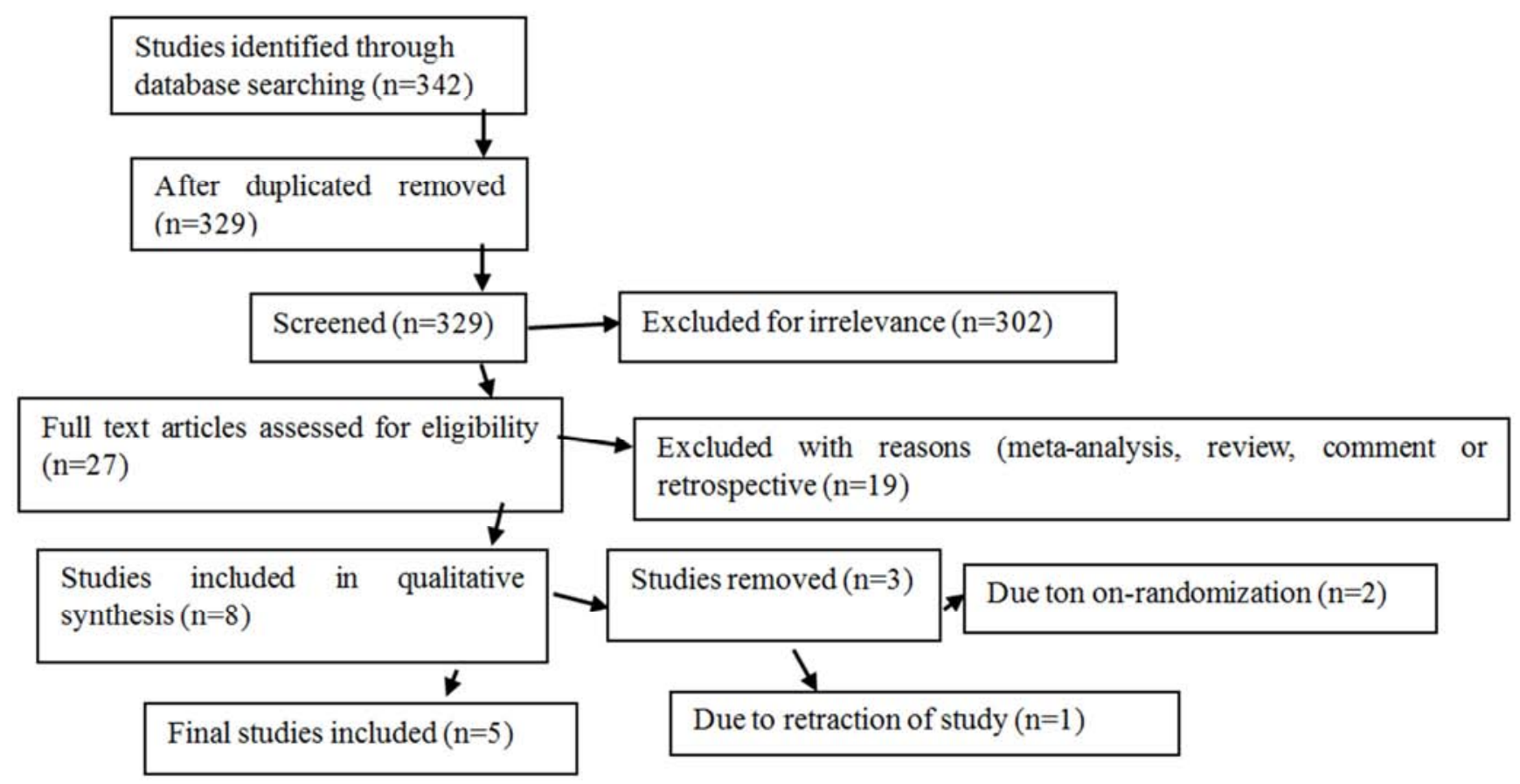

Figure 1. Prisma. 


\subsection{Data Collection and Statistical Analysis}

All analyses were performed using Comprehensive MetaAnalysis V3 (Trial) software. The meta-analysis was performed according to the recommendations of The Cochrane Collaboration and the Cochrane Handbook for Systematic Reviews of Interventions [25].

The summarized statistics of categorical outcomes are presented as risk ratios for complications and treatment efficacy. Risk ratio was preferred to odds ratio with $95 \%$ confidence intervals (CIs), as the latter is more appropriate for case-control studies. The Mantel-Haenszel method was used to combine the summarized statistics and assess the heterogeneity between studies by considering the " $I^{2}$ ", method alongside the chi-square $P$ value. Interpretation of the $I^{2}$ statistic was based on the guidelines in the Cochrane Handbook, in which $0 \%$ to $40 \%$ may be unimportant heterogeneity, $30 \%-60 \%$ indicates moderate, $50 \%-90 \%$ indicates substantial, and $75 \%-100 \%$ indicates considerable heterogeneity. When combining studies of this nature for meta-analysis, we assumed that variation existed between trials in both the design and the methodological quality, so we used a random effect model to provide a conservative estimate of the results. A fixed effect model was used when no heterogeneity existed.

Continuous variables, when the mean and SD were presented, were assessed using the weighted mean difference
(WMD) with the $95 \% \mathrm{CI}$ and a random-effects model was used according to heterogeneity if significant heterogeneity was present. We present the results of statistical heterogeneity in the forest plots. We considered the results to be statistically significant at the $P$ value less than 0.05 if the $95 \%$ confidence interval did not include the value "one" for risk ratio or the value "zero" for WMD.

\section{Results}

The PubMed, Medline, Medscape and Cochrane online library databases yielded 329 possibly relevant studies after duplicates removed. We excluded 321 papers, of which 302 were irrelevant and nineteen were meta-analyses, reviews, comments or retrospective studies. After further assessment, two out of the eight studies remaining were excluded due to non-randomization. A final study authored by Malik et al. [26] was excluded because it was retracted.

Five prospective RCTs [27-31] with a total of 1430 patients met the selection criteria and were eligible for inclusion. The number of patients, per protocol, allocated to the antibiotic group was 644 , while 786 patients comprised the surgery group.

The characteristics of the studies included are summarized in Table 1. The treatment efficacy parameters dictated by each study is included in Table 2 .

Table 1. Characteristics of included studies.

\begin{tabular}{lllllll}
\hline & Males/Females & Age & No. of Pts. in AG & No. of Pts. in SG & Methods of diagnosis & Treated successfully in AG \\
\hline Erikson et al. (1995) & $27 / 13$ & $18-75$ & 20 & 20 & US, Chemistry & $12(60 \%)$ \\
Styrud et al. (2006) & $252 / 0$ & $18-50$ & 128 & 124 & US, Chemistry & $113(88.3 \%)$ \\
Hansson et al. (2009) & $195 / 174$ & $>18$ & 202 & 167 & US/CT, Chemistry & $83(41.1 \%)$ \\
Hansson et al. (2009) & $200 / 169$ & $>18$ & 119 & 250 & US/CT, Chemistry & $93(78.2 \%)$ \\
Vons et al. (2011) & $143 / 96$ & $18-68$ & 120 & 119 & CT, Chemistry & $81(67.5 \%)$ \\
Salinen et al. (2015) & $329 / 201$ & $18-60$ & 257 & 273 & CT & $186(72.4 \%)$ \\
Total & & & 644 & 786 & & $485(75.3 \%)$ \\
\hline
\end{tabular}

Table 1. Continue.

\begin{tabular}{lllllll}
\hline & $\begin{array}{l}\text { Normal appendix } \\
\text { in SG }\end{array}$ & $\begin{array}{l}\text { Hospital stay in } \\
\text { SG (days) }\end{array}$ & $\begin{array}{l}\text { Hospital stay in } \\
\text { AG (days) }\end{array}$ & $\begin{array}{l}\text { Follow-up period } \\
\text { (months) }\end{array}$ & $\begin{array}{l}\text { No. of Pts. lost to } \\
\text { follow-up in AG }\end{array}$ & $\begin{array}{l}\text { No. of Pts. lost to } \\
\text { follow-up in AG }\end{array}$ \\
\hline Erikson et al. (1995) & 3 & 3.1 & 3.4 & 1 & 0 & 1 \\
Styrud et al. (2006) & 4 & 3 & 2.6 & 12 & 0 & 0 \\
Hansson et al. (2009) & 2 & 2 & 3 & 12 & 10 & 28 \\
Hansson et al. (2009) & 20 & 2 & 3 & 12 & 10 & 28 \\
Vons et al. (2011) & 3 & 3.96 & 3.04 & 12 & 9 & 15 \\
Salinen et al. (2015) & 2 & 3 & 3 & & 30 & 58 \\
Total & $42(5.3 \%)$ & & & & $49(7.6 \%)$ & $102(13 \%)$ \\
\hline
\end{tabular}

AG: antibiotic group

SG: surgery group

1: Intention-to-treat

2: per-protocol

US: ultrasound

CT: computerized tomography

*: Total includes Hansson et al.'s per-protocol results only. 
Table 2. Treatment efficacy parameters.

\begin{tabular}{lll}
\hline & Treatment efficacy parameters in antibiotic group & Treatment efficacy parameters in surgery group \\
\hline Erikson et al. (1995) & Decrease in pain using visual analogue scale, WBC levels, CRP & Decrease in pain using visual analogue scale, WBC levels, \\
values & CRP values \\
Styrud et al. (2006) & Improvement in symptoms with no local peritonitis & N/A \\
Hansson et al. (2009) & $\begin{array}{l}\text { Definite improvement without the need for surgery within a } \\
\text { median follow-up of one year }\end{array}$ & $\begin{array}{l}\text { Confirmed appendicitis at operation or another appropriate } \\
\text { surgical indication for operation }\end{array}$ \\
Vons et al. (2011) & $\begin{array}{l}\text { Occurrence of peritonitis within 30 days } \\
\text { Discharge from the hospital without the need for surgery and no } \\
\text { Salinen et al. (2015) }\end{array}$ & $\begin{array}{l}\text { Occurrence of peritonitis within 30 days } \\
\text { recurrent appendicitis during 1 year follow up period }\end{array}$ \\
\hline
\end{tabular}

CRP: c-reactive protein

The total number of complications in the antibiotic group is $3.6 \%$ which is significantly lower than that observed in the surgery group; $11.6 \%$. However, when taking recurrence rate into account, the overall failure rate of antibiotic treatment reaches $21.9 \%$ (Table 3 ).

Heterogeneity is found for the prevalence of thirty-day post- therapeutic peritonitis $\left(I^{2}=79.195 \%, P=0.002\right)$. Because of this we used random model results. The prevalence of "thirtyday post-therapeutic peritonitis" was similar in both groups according to the random model $(\mathrm{MH}$ risk ratio $=3.216,95 \%$ CI; 0.530-19.514, $P=0.204$ ) (Table 4).

Table 3. Unsuccessful treatment in both groups.

\begin{tabular}{|c|c|c|c|c|}
\hline & $\begin{array}{l}\text { Number of recurrent } \\
\text { cases in antibiotic group }\end{array}$ & $\begin{array}{l}\text { Number of complications } \\
\text { in antibiotic group }\end{array}$ & $\begin{array}{l}\text { Antibiotic Failure } \\
\text { (recurrences + complications) }\end{array}$ & $\begin{array}{l}\text { Number of complications } \\
\text { in surgery group }\end{array}$ \\
\hline Erikson et al. (1995) & $7(35 \%)$ & $1(5 \%)$ & $8(40 \%)$ & $2(10 \%)$ \\
\hline Styrud et al. (2006) & $16(12.5 \%)$ & $4(3.1 \%)$ & $20(15.6 \%)$ & $17(13.7 \%)$ \\
\hline Hansson et al. (2009) ${ }^{1}$ & $14(6.9 \%)$ & $11(5.4 \%)$ & $25(12.4 \%)$ & $18(10.8 \%)$ \\
\hline Hansson et al. $(2009)^{2}$ & $15(12.6 \%)$ & $3(2.5 \%)$ & $18(15.1 \%)$ & $25(10 \%)$ \\
\hline Vons et al. (2011) & $30(25 \%)$ & $9(7.5 \%)$ & $39(32.5 \%)$ & $2(1.7 \%)$ \\
\hline Salinen et al. (2015) & $50(19.5 \%)$ & $6(2.3 \%)$ & $56(21.8 \%)$ & $45(16.5 \%)$ \\
\hline Total $^{*}$ & $118(18.3 \%)$ & $23(3.6 \%)$ & $141(21.9 \%)$ & $91(11.6 \%)$ \\
\hline
\end{tabular}

1: Intention-to-treat

2: per-protocol

*: Total includes Hansson et al.’s per-protocol results only

Table 4. Results for the prevalence of thirty-day post-therapeutic peritonitis.

\begin{tabular}{|c|c|c|c|c|c|c|c|c|c|c|c|c|c|}
\hline \multirow{2}{*}{ Model } & \multirow{2}{*}{ Study Name } & \multicolumn{5}{|c|}{ Statistics for each study } & \multicolumn{2}{|c|}{ Events / Total } & \multicolumn{5}{|c|}{ MH risk ratio and $95 \% \mathrm{CI}$} \\
\hline & & MH risk ratio & LL & UL & Z-value & P-value & Antibiotic & Surgery & 0.01 & 0.1 & 1 & 10 & 100 \\
\hline & Erikson et al. (1995) & 3 & 0.129 & 69.52 & 0.69 & 0.493 & $1 / 20$ & $0 / 20$ & & & & & \\
\hline & Styrud et al. (2006) & 30.1 & 1.817 & 496.6 & 2.38 & 0.017 & $15 / 128$ & $0 / 124$ & & & & & \\
\hline & Hansson et al. (2009) & 0.756 & 0.528 & 1.083 & -1.52 & 0.128 & $43 / 202$ & $47 / 167$ & & & & & \\
\hline & Vons et al. (2011) & 4.463 & 0.985 & 20.22 & 1.94 & 0.052 & $9 / 120$ & $2 / 119$ & & & & & \\
\hline Fixed & & 1.187 & 0.859 & 1.639 & 1.04 & 0.300 & & & & & & & \\
\hline Random & & 3.216 & 0.530 & 19.51 & 1.27 & 0.204 & & & & & & - & \\
\hline
\end{tabular}

MH: Mantel-Haenszel; LL: Lower Level; UL: Upper Level; CI: Confidence Interval; I-Square=79.195 (P=0.002)

The prevalence of "Normal appendix" was similar in both groups according to the random model $(\mathrm{MH}$ risk ratio $=2.964$ 95\% CI; 0.901-9.748, $P=0.074)$. Heterogeneity was not found in the studies $\left(I^{2}=0.648 \%, P=0.389\right)$ (Table 5).

The prevalence of "total complications" was similar in both groups according to the random model $(\mathrm{MH}$ risk ratio $=0.442$, 95\% CI; 0.178-1.101, $P=0.080)$. Heterogeneity is found in studies $\left(I^{2}=79.256 \%, P=0.001\right)$ (Table 6).

The overall difference for length of hospital stay between antibiotic therapy and surgery is not found to be statistically significant (95\% CI): $0.184(-0.120,0.488) P=0.236]$. Heterogeneity is found in the studies $\left(\mathrm{I}^{2}=40.011 \%, P=0.155\right)($ Table 7$)$.

Table 5. Results for the prevalence of normal appendix.

\begin{tabular}{|c|c|c|c|c|c|c|c|c|c|c|c|c|c|}
\hline \multirow{2}{*}{ Model } & \multirow{2}{*}{ Study Name } & \multicolumn{5}{|c|}{ Statistics for each study } & \multicolumn{2}{|c|}{ Events / Total } & \multicolumn{5}{|c|}{ MH risk ratio and $95 \% \mathrm{CI}$} \\
\hline & & MH risk ratio & $\mathbf{L L}$ & $\mathbf{U L}$ & Z-value & P-value & Antibiotic & Surgery & 0.01 & 0.1 & 1 & 10 & 100 \\
\hline & Erikson et al. (1995) & 7 & 0.385 & 127.3 & 1.32 & 0.189 & $3 / 20$ & $0 / 20$ & & & & & \\
\hline & Styrud et al. (2006) & 0.323 & 0.013 & 7.85 & -0.69 & 0.488 & $0 / 128$ & $1 / 124$ & & & & & \\
\hline & Vons et al. (2011) & 10.9 & 0.610 & 195.1 & 1.62 & 0.104 & $5 / 120$ & $0 / 119$ & & & & & \\
\hline & Salminen et al. (2015) & 2.656 & 0.520 & 13.56 & 1.17 & 0.241 & $5 / 257$ & $2 / 273$ & & & & & \\
\hline Fixed & & 3.274 & 1.147 & 9.35 & 2.22 & 0.027 & & & & & & & \\
\hline Random & & 2.964 & 0.901 & 9.75 & 1.79 & 0.074 & & & & & & & \\
\hline
\end{tabular}

MH: Mantel-Haenszel; LL: Lower Level; UL: Upper Level; CI: Confidence Interval; I-Square=0.648 (P=0.389) 
Table 6. Results for the prevalence of total complications.

\begin{tabular}{|c|c|c|c|c|c|c|c|c|c|c|c|c|c|}
\hline \multirow{2}{*}{ Model } & \multirow{2}{*}{ Study Name } & \multicolumn{5}{|c|}{ Statistics for each study } & \multicolumn{2}{|c|}{ Events / Total } & \multicolumn{5}{|c|}{ MH risk ratio and $95 \% \mathrm{CI}$} \\
\hline & & MH risk ratio & $\mathbf{L L}$ & $\mathbf{U L}$ & Z-value & P-value & Antibiotic & Surgery & 0.01 & 0.1 & 1 & 10 & 100 \\
\hline & Erikson et al. (1995) & 0.200 & 0.010 & 3.920 & -1.06 & 0.289 & $0 / 20$ & $2 / 20$ & & & & & \\
\hline & Styrud et al. (2006) & 0.342 & 0.139 & 0.839 & -2.44 & 0.019 & $6 / 128$ & $17 / 124$ & & & & & \\
\hline & Hansson et al. (2009) & 0.767 & 0.556 & 1.057 & -1.62 & 0.105 & $51 / 202$ & $55 / 167$ & & & & & \\
\hline & Vons et al. (2011) & 1.653 & 0.404 & 6.761 & 0.70 & 0.485 & $5 / 120$ & $3 / 119$ & & & & & \\
\hline & Salminen et al. (2015) & 0.142 & 0.061 & 0.326 & -4.59 & 0.001 & $6 / 257$ & $45 / 273$ & & & & & \\
\hline Fixed & & 0.503 & 0.383 & 0.661 & -4.95 & 0.001 & & & & & & & \\
\hline Random & & 0.442 & 0.178 & 1.101 & -1.75 & 0.080 & & & & & & & \\
\hline
\end{tabular}

MH: Mantel-Haenszel; LL: Lower Level; UL: Upper Level; CI: Confidence Interval; I-Square=79.26 (P=0.001)

Table 7. Results for the prevalence of length of hospital stay (days).

\begin{tabular}{|c|c|c|c|c|c|c|c|c|c|c|c|c|c|c|}
\hline \multirow{2}{*}{ Model } & \multirow{2}{*}{ Study Name } & \multicolumn{6}{|c|}{ Statistics for each study } & \multicolumn{2}{|c|}{ Sample Size } & \multicolumn{4}{|c|}{$\begin{array}{l}\text { Difference in means and } \\
95 \% \text { CI }\end{array}$} & \\
\hline & & $\begin{array}{l}\text { Difference } \\
\text { in means }\end{array}$ & $\begin{array}{l}\text { Standar } \\
\text { d error }\end{array}$ & LL & UP & Z-value & P-value & Antibiotic & Surgery & -1 & -0.5 & $\mathbf{0}$ & 0.5 & \\
\hline \multirow[b]{6}{*}{ Fixed } & Erikson et al. (1995) & -0.30 & 0.425 & -1.13 & 0.53 & -0.71 & 0.481 & 20 & 20 & & & & 一 & \\
\hline & Styrud et al. (2006) & 0.40 & 0.164 & 0.08 & 0.72 & 2.43 & 0.015 & 128 & 124 & & & & & \\
\hline & Hansson et al. (2009) & 0.00 & 0.227 & -0.45 & 0.45 & 0.00 & 1.000 & 202 & 167 & & & & & \\
\hline & Vons et al. (2011) & 0.92 & 0.467 & 0.01 & 1.84 & 1.97 & 0.049 & 120 & 119 & & & & & \\
\hline & Salminen et al. (2015) & 0.00 & 0.246 & -0.48 & 0.48 & 0.00 & 1.000 & 257 & 273 & & & & & \\
\hline & & 0.21 & 0.110 & -0.01 & 0.42 & 1.90 & 0.057 & & & & & & & \\
\hline Random & & 0.18 & 0.155 & -0.12 & 0.49 & 1.19 & 0.236 & & & & & & & \\
\hline
\end{tabular}

LL: Lower Level; UL: Upper Level; CI: Confidence Interval; I-Square=79.26 ( $\mathrm{P}=0.001)$

The overall difference between antibiotic therapy and surgery is not statistically significant in terms of mean duration of pain (Difference in means $=-1.170$, standard error $=1.398, P=0.403)($ Table 8$)$.

Regarding the overall mean duration of disability, the antibiotic group has a statistically significant shorter duration of disability than that of the surgery group $(P=0.047)$. Heterogeneity is not found in these studies $\left(I^{2}=0 \%, P=\right.$ 0.348) (Table 9).

Table 8. Results for the prevalence of mean duration of pain.

\begin{tabular}{|c|c|c|c|c|c|c|c|c|c|c|c|c|c|c|}
\hline \multirow{2}{*}{ Model } & \multirow{2}{*}{ Study Name } & \multicolumn{6}{|c|}{ Statistics for each study } & \multicolumn{2}{|c|}{ Sample Size } & \multicolumn{4}{|c|}{$\begin{array}{l}\text { Difference in means and } \\
95 \% \text { CI }\end{array}$} & \\
\hline & & $\begin{array}{l}\text { Difference } \\
\text { in means }\end{array}$ & $\begin{array}{l}\text { Standard } \\
\text { error }\end{array}$ & $\mathbf{L L}$ & UP & Z-value & P-value & Antibiotic & Surgery & -1 & -0.5 & $\mathbf{0}$ & 0.5 & 1 \\
\hline & Erikson et al. (1995) & 2.60 & 4.215 & 17.7 & -5.66 & 0.62 & 0.537 & 20 & 20 & & & & & \\
\hline & Hansson et al. (2009) & -3.00 & 0.105 & 0.01 & -3.21 & -28.6 & 0.001 & 202 & 167 & & & & & \\
\hline & Vons et al. (2011) & -0.07 & 0.158 & 0.03 & -0.38 & -0.44 & 0.657 & 120 & 119 & & & & & \\
\hline Fixed & & -2.10 & 0.087 & 0.01 & -2.27 & -24.1 & 0.001 & & & & & & & \\
\hline
\end{tabular}

LL: Lower Level; UL: Upper Level; CI: Confidence Interval; I-Square=99.17 ( $\mathrm{P}=0.0001)$

Table 9. Results for the prevalence of duration of disability.

\begin{tabular}{|c|c|c|c|c|c|c|c|c|c|c|c|c|c|c|}
\hline \multirow{2}{*}{ Model } & \multirow{2}{*}{ Study Name } & \multicolumn{6}{|c|}{ Statistics for each study } & \multicolumn{2}{|c|}{ Sample Size } & \multicolumn{5}{|c|}{$\begin{array}{l}\text { Difference in means and } \\
95 \% \mathrm{CI}\end{array}$} \\
\hline & & $\begin{array}{l}\text { Difference } \\
\text { in means }\end{array}$ & $\begin{array}{l}\text { Standard } \\
\text { error }\end{array}$ & $\mathbf{L L}$ & $\mathbf{U P}$ & Z-value & P-value & Antibiotic & Surgery & -8 & -4 & $\mathbf{0}$ & 4 & 8 \\
\hline & Stryud et al. (2006) & -2.10 & 0.98 & -4.03 & -0.17 & -2.14 & 0.033 & 128 & 124 & & & & & \\
\hline & Vons et al. (2011) & -0.63 & 1.22 & -3.02 & 1.76 & 0.52 & 0.606 & 120 & 119 & & & & & \\
\hline Fixed & & -1.52 & 0.77 & -3.02 & -0.02 & -1.98 & 0.047 & & & & & & & \\
\hline Random & & -1.52 & 0.76 & -3.02 & -0.02 & -1.98 & 0.047 & & & & & & & \\
\hline
\end{tabular}

LL: Lower Level; UL: Upper Level; CI: Confidence Interval; I-Square $=0.00(\mathrm{P}=0.348)$

\section{Discussion}

In this meta-analysis, a new and well-designed study published in 2015 [27] is taken into account. This study, by Salminen et al., involved the largest sample of patients of all the discussed studies, making it more representative of the general population. In addition, a handful of outcomes are discussed in order to present a more comprehensive comparison of the treatment modalities.

Each of the selected studies adopted a different set of parameters to consider the treatment successful. 
Improvement in symptoms, mainly pain, is used as an indicator for treatment efficacy in three studies. Two of these also used normal laboratory investigations, such as CRP and WBC count in addition to the decrease in the visual analogue scale as parameters. Lack of need for surgery throughout the follow-up period is another parameter used by both Hansson et al. and Salminen et al. As for surgery, success is mainly dependent on the completion of appendectomy and confirmation of appendicitis at operation, without the occurrence of peritonitis within thirty days (Table 2).

The success of antibiotic treatment for acute appendicitis ranges from $60 \%$ to $88.3 \%$ according to the randomized controlled trials at hand. As evident from this wide range, many variables can affect treatment efficacy including the definition of successful treatment, accuracy of diagnosis, type of antibiotics used and duration of follow up. A mean of $65.3 \%$ of patients allocated to the antibiotic group, perintention-to-treat, were treated successfully. However, if the same parameter is calculated using the per-protocol values in the Hansson et al. study, the success rate of antibiotic treatment reaches $75.3 \%$. Thus, values for complication and recurrence rates are calculated depending on per-protocol values only to avoid crossover of patients and to eliminate any failures that might have been successes had the involved physicians continued with the regimen dictated by the intention-to-treat groups. It is also observed that the $17.8 \%$ who presented with recurrent appendicitis after antibiotic treatment showed no chronic findings upon histopathological examination. Although the difference in the number of total complications between the two groups is statistically insignificant $(p=0.08)$, studies coincide that complications seen in patients allocated to the antibiotic group are less severe than those seen in the surgical group. Most complications seen in patients undergoing appendectomy were major, such as abscesses, small bowel obstruction, wound rupture and pulmonary embolism. As for patients allocated to the antibiotic group, complications were relatively minor including fungal infection, diarrhea and bladder dysfunction.

Three out of the fifteen patients who had recurrent appendicitis in the study conducted by Hansson underwent a second round of antibiotics, which led to complete resolution of the condition with no recurrence or complications in a one-year follow-up period. Although the patients with recurrent appendicitis in the Styrud et al. study underwent surgery, most of them requested to be treated with another course of antibiotics, indicating high patient satisfaction. Moreover, Eriksson et al. stated that patients given antibiotics before surgery did not have any postoperative infection. This suggests that antibiotic use can eliminate some of the complications associated with surgery and, possibly, delay surgery in uncomplicated cases.

In spite of having the same length of hospital stay and duration of pain as the surgery group (Table 7 and 8), patients in the antibiotic group have a shorter duration of disability and are able to return to work earlier than their counterparts who underwent appendectomy (Table 9). These patients also have a lower, faster declining WBC count, lower body temperatures and decreased incidence of local peritonitis. It can be also inferred that antibiotics significantly reduce the risks associated with surgery and eliminate those associated with anesthesia, which are a possibility in patients undergoing appendectomy. The study conducted by Hansson et al. showed that major complications found in the surgery group were three-fold those observed in the antibiotic group, with comparable conclusions in the other studies.

The selected studies used similar antibiotic regimens in the treatment of the conservative groups. During the hospital stay, which ranged from one to three days, three studies used a third generation cephalosporin, namely cefotaxime, in combination with either metronidazole or tinidazole. Vons et al. chose to administer amoxicillin and clavulinic acid to the antibiotic group. The decision was based on the findings of an earlier study, which proved that this combination is superior to other antibiotics when used in uncomplicated sigmoid diverticulitis [32]. However, it is worth noting that E. coli's resistance to amoxicillin and clavulinic acid has been increasing over the past few years [33]. This might account for the high recurrence rate $(25 \%)$ seen in this particular study. The most recent RCTs used ertapenem, following the previously published guidelines by the surgical infection society and the infectious diseases society of America for the management of intra-abdominal infection [34]. As for post-discharge antibiotics, four of the five selected studies used a combination of a second or third generation fluoroquinolone; ofloxacin, ciprofloxacin or levofloxacin, and a nitroimidazole; metronidazole or tinidazole [27-29, 31]. Vons et al. continued to use amoxicillin and clavulinic acid. These post-discharge antibiotics were given for a period ranging from 7 to 10 days. Therefore, it is worth mentioning that there is no consensus on the type of antibiotic to be used and is yet to be agreed upon.

Three of the studies did not use CT for diagnosis of acute appendicitis, inadvertently increasing the percentage of negative appendectomies. In Vons et al., and Salminen et al., CT was the main method of diagnosis, reducing the rate of negative appendectomy to $1.3 \%$, compared to $9.4 \%$ in the studies that did not use CT for diagnosis. It has been suggested by Vons et al. that complications seen in patients who underwent appendectomy after failure of antibiotic treatment might have been already present and missed at the time of diagnosis. This is also supported by the fact that all the complications in the antibiotic group in Salminen et al.'s study were found during the primary hospital stay, while none were found in later recurring cases. Surgical complications as well as failure of antibiotics treatment were also thought to be associated with the presence of a stercolith in the appendix on CT. This further enhances the importance of CT scans for correct status evaluation; complicated versus uncomplicated, and consequently, for choosing whether patients are to be treated conservatively or operatively. A notable drawback of CT utilization would be the delay in antibiotic administration, ultimately leading to decreased efficacy.

While emergency appendectomy, for the time being, 
remains the gold standard for treating acute appendicitis, the use of antibiotics can be seen as a reliable substitution in certain circumstances to reduce the risks associated with surgery. In addition, antibiotics can also be safely recommended for elderly, patients with comorbidities and obese patients instead of surgery, as operative morbidity is higher in these cases.

According to the data collected, $75.3 \%$ of acute appendicitis cases have proven to be successfully treated by antibiotics alone. The remaining $24.7 \%$ of cases, in which antibiotics were unsuccessful, raise the doubt that the etiologies of these cases may differ from those in which antibiotics were successful.

\section{Conclusion}

It is of major importance to know why antibiotic treatment fails in some cases. Therefore, we emphasize the need for upcoming studies to focus particularly on this topic. This may subsequently enable patient-targeted therapy according to the exact pathophysiology of acute appendicitis.

\section{References}

[1] Fitz RH (1886) Perforating inflammation of the vermiform appendix. Am J Med Sci 92: 321-46.

[2] Humes DJ, Simpson J (2006) Acute appendicitis. BMJ 333: $530-4$.

[3] Addiss DG, Shaffer N, Fowler BS, et al. (1990) The epidemiology of appendicitis and appendectomy in the United States. Am J Epidemiol 132(5): 910-25.

[4] Mc Burney C (1889) Experience with early operative interference in cases of disease of the vermiform appendix. $\mathrm{N}$ Y Med J 50: 676-84.

[5] Blomqvist P, Ljung H, Nyren O, et al. (1998) Appendectomy in Sweden 1989-1993 assessed by the Inpatient Registry. J Clin Epidemiol 51: 859-65.

[6] O'Connell PR, Williams NS, Bulstrode CJK (2008) The vermiform appendix Bailey and Love's short practice of surgery. 25th ed. p. 1204-18.

[7] Margenthaler JA, Longo WE, Virgo KS, et al. (2003). Risk factors for adverse outcomes after the surgical treatment of appendicitis in adults. Ann Surg 238: 59-66.

[8] Hale DA, Molloy M, Pearl RH, et al. (1997) Appendectomy: a contemporary appraisal. Ann Surg 225: 252-61.

[9] Colson M, Skinner KA, Dunnington G (1997) High negative appendectomy rates are no longer acceptable. Am J Surg 174: 723-6; discussion 726-7.

[10] Guller U, Jain N, Peterson ED, et al. (2004) Laparoscopic appendectomy in the elderly. Surgery 135: 479-88.

[11] Nakhamiyayev V, Galldin L, Chiarello M, et al. (2009) Laparoscopic appendectomy is the preferred approach for appendicitis: a retrospective review of two practice patterns. Surg Endosc 24(4): 859-864.
[12] Sauerland S, Lefering R, Neugebauer EAM. Laparoscopic versus open surgery for suspected appendicitis. Cochrane Database of Systematic Reviews Issue 10.

[13] Harrison, Paul W (1953) Appendicitis and the Antibiotics. Am J Surg 85.2: 160-63.

[14] Coldrey, E (1956) Treatment of Acute Appendicitis. BMJ 2.5007: $1458-461$.

[15] No authors listed (1979) Treatment of Acute Abdominal Diseases by Combined Traditional Chinese and Western Medicine. World J Surg 3.1: 91-94.

[16] Whetsone D, Hazey J, Pofahl WE 2nd, et al. (2004) Current management of diverticulitis. Curr Surg 61: 361-365.

[17] Livingston, Edward H (2011) "Epidemiological Similarities between Appendicitis and Diverticulitis Suggesting a Common Underlying Pathogenesis." Arch Surg 146.3: 308.

[18] Adams ML (1990) The medical management of acute appendicitis in a nonsurgical environment: A retrospective case review. Mil Med 155: 345-347.

[19] Liu K, Ahanchi S, Pisaneschi M, et al. (2007) Can acute appendicitis be treated by antibiotics alone? Am Surg 73: $1161-1165$.

[20] Winn RD, Laura S, Douglas C, et al. (2004) Protocol-based approach to suspected appendicitis, incorporating the Alvarado score and outpatient antibiotics. NZJ Surg 74: 324 329.

[21] Liu K, Fogg L (2011) Use of antibiotics alone for treatment of uncomplicated acute appendicitis: a systematic review and meta-analysis. Surgery 150: 673-83.

[22] Ansaloni L, Catena F, Coccolini F, et al. (2011) Surgery versus conservative antibiotic treatment in acute appendicitis: a systematic review and meta-analysis of randomized controlled trials. Dig Surg 28.3: 210-21

[23] Kirby A, Hobson RP, Burke D, et al. (2015) Appendectomy for suspected uncomplicated appendicitis is associated with fewer complications than conservative antibiotic management: a meta-analysis of post-intervention complications. J Infect. 70: $105-10$

[24] Varadhan KK, Humes DJ, Neal KR, et al. (2010) Antibiotic therapy versus appendectomy for acute appendicitis: a metaanalysis. World J Surg. 34: 199-209.

[25] Higgins JPT, Green SM (2011) Cochrane handbook for systematic reviews of interventions. Copenhagen, Denmark.

[26] Malik AA, Bari S-U. RETRACTED ARTICLE (2009) Conservative Management of Acute Appendicitis. J Gastrointest Surg 13(5): 966-70.

[27] Salminen P, Paajanen H, Rautio T, et al. (2015) Antibiotic Therapy vs Appendectomy for Treatment of Uncomplicated Acute Appendicitis. Jama 313(23): 2340.

[28] Eriksson S, Granström L (1995) Randomized controlled trial of appendicectomy versus antibiotic therapy for acute appendicitis. Br J Surg 82(2): 166-9.

[29] Hansson J, Körner U, Khorram-Manesh A, et al. (2009) Randomized clinical trial of antibiotic therapy versus appendicectomy as primary treatment of acute appendicitis in unselected patients. Br J Surg 96(5): 473-81. 
[30] Vons C, Barry C, Maitre S, et al. (2011) Amoxicillin plus clavulanic acid versus appendectomy for treatment of acute uncomplicated appendicitis: an open-label, non-inferiority, randomised controlled trial. The Lancet 377(9777): 1573-9.

[31] Styrud J, Eriksson S, Nilsson I, et al. (2006) Appendectomy versus Antibiotic Treatment in Acute Appendicitis. A Prospective Multicenter Randomized Controlled Trial. World J Surg 30(6): 1033-7.

[32] Schug-Pass C, Geers P, Hügel O, et al. (2010) Prospective randomized trial comparing short-term antibiotic therapy versus standard therapy for acute uncomplicated sigmoid diverticulitis. Int J Colorectal Dis 25(6): 751-59.

[33] Oteo J, Campos J, Lázaro E, et al. (2008) Increased Amoxicillin-Clavulanic Acid Resistance in Escherichia coli Blood Isolates. Emerg Infect Dis 14(8): 1259-62.

[34] Solomkin JS, Mazuski JE, Beadley JS, et al. (2010) Diagnosis and management of complicated intra-abdominal infection in adults and children: Guidelines by the Surgical Infection Society and the Infectious Diseases Society of America. Clin Infect Dis 50(2): 133-64. 